\title{
Photoacid Generation in Polymer Solids Induced and Observed with Near-Field Scanning Optical Microscopy
}

\author{
Satoshi Takahashi*, Shinjiro Machida, and Kazuyuki Horie
}

\author{
Department of Chemistry and Biotechnology, Graduate School of Engineering, \\ The University of Tokyo \\ 7-3-1 Bunkyo, Hongo, Tokyo 113-8656, JAPAN
}

\begin{abstract}
Near-field scanning optical microscopy (NSOM) has been used both to induce and to observe acid generation from a photoacid generator (PAG) doped in poly(methylmethacrylate) (PMMA) films. As a PAG, p-nitrobenzyl 9,10-dimethoxyanthracene-2-sulfonate (NAS) is used. Acid from PAG is monitored by fluorescence from a $\mathrm{pH}$ sensitive dye, Nile blue A (NBA). Our NSOM has a spatial resolution of $100 \mathrm{~nm}$ which is evaluated from the measurements of a chromium test pattern. A 442 $\mathrm{nm}$ radiation from an NSOM probe is used for inducing photoacid generation, and a $660 \mathrm{~nm}$ fluorescence from NBA cations which is excited with a $620 \mathrm{~nm}$ light passing through the NSOM probe is used for imaging the spatial distribution of the photoacid. The photoacid-generated area is successfully observed using fluorescence from NBA cations. Comparison between the topographic and NSOM image reveals that NSOM fluorescence image is not affected by the topographic features which are not formed by photochemical reactions.
\end{abstract}

Keywords:near-field scanning optical microscopy (NSOM), acid diffusion, photoacid generator (PAG), 9,10-dimethoxyanthracene-2-sulfonate (NAS), Nile blue A (NBA)

\section{Introduction}

Chemical amplification is an important chemical reaction in deep-UV lithography process in the semiconductor industry, because polymeric photoresists have high optical densities in deep-UV region, leading to a low sensitivity to UV irradiation [1,2].

Chemical amplification is a catalytic process in which protons generated by photochemical processes act as a catalyst, which compensates the low transparency of the polymer photoresists to UV light. Materials which generate acid during photolithography process are called photoacid generators (PAGs). The quality of the patterns made in chemically amplified photoresists is greatly affected by the photochemical processes and diffusion of PAG. Therefore monitoring microscopic behavior of the photoacid generation in polymer solids is important in order to develop better photoresists for higher density patterning.

A spectroscopic technique was previously proposed by Yamakawa et al. [3], where the diffusion of the photoacid was analyzed using fluorescence from a pH sensitive dye, Nile Blue A (NBA), doped together with the PAG. The basic form of NBA is so reactive with acidic substances that its protonation proceeds irreversibly resulting in NBA cation except under basic condition [4]. Using this method, diffusion coefficients of the acids in the polymer solids were quantitatively evaluated. This study was based on a macroscopic

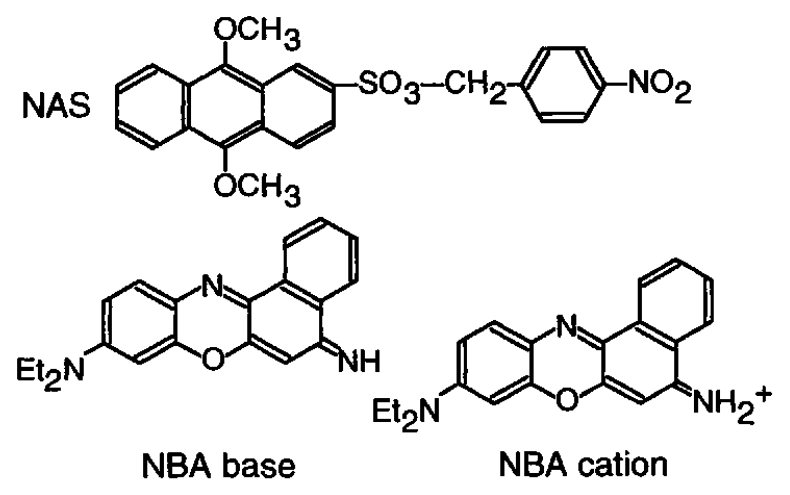

Figure 1 Chemical structures of NAS and basic and cationic forms of NBA. 


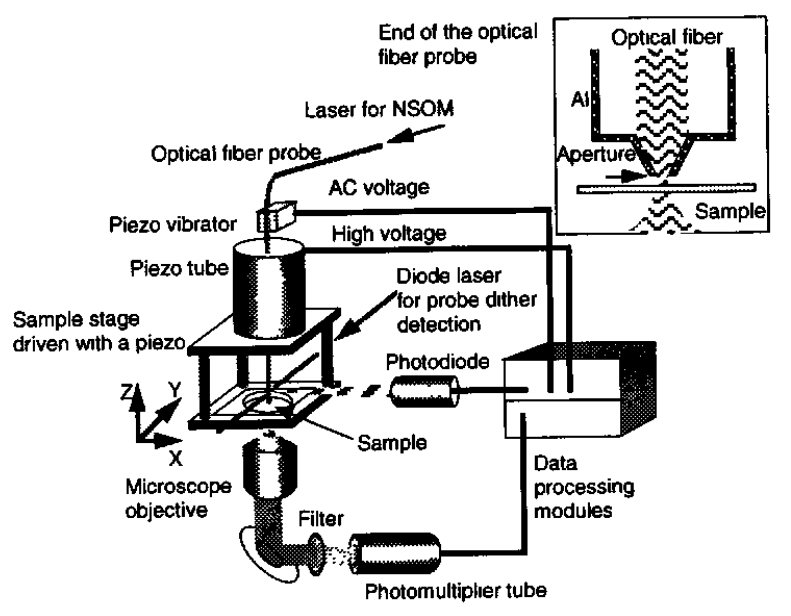

Figure 2 Schematic illustration of NSOM

spectroscopy. If microscopic patterning and observation for chemically amplificated photoresists are performed, they would provide much information of the photoacid generation and diffusion of photoacids in the small areas in the photoresists.

Near-field scanning optical microscopy (NSOM) is a viable technique to study photochemistry in small areas, because it can produce optical images with a spatial resolution beyond the diffraction limit $[5,6]$. NSOM employs a subwavelength-sized light source which is placed in close proximity to the sample surface and is scanned across it. The light passing through the NSOM probe interacts with the sample surface before it diffracts out, therefore the spatial resolution of the obtained optical images is as high as the size of the light source, not dominated by the diffraction limit. Owing to its super resolution, and combined with a technique of single molecule detection, NSOM has become an admirable tool for investigating molecular diffusion in polymers $[7,8]$. Not only as a tool of image acquisition, NSOM can be used to induce photochemical reactions in very small areas [9]. In a previous work [10], NSOM using micropipettes was applied to the study of photoacid generation in polymer solids, and it was demonstrated that the photoacid generation is successfully induced by light passing through the NSOM probe. In the present study a mechanism for regulating tip-sample distance is implemented, and sharpened optical fibers are employed as NSOM probes. Owing to these modifications, photoacid generation in a polymer solid is induced and observed with NSOM and the obtained NSOM images are discussed compared with the corresponding topographic image.

\section{Experimental}

As a PAG, p-nitrobenzyl 9,10dimethoxyanthracene-2-sulfonate (NAS) was used [11]. The photoacid from NAS was monitored by the fluorescence from a $\mathrm{pH}$ sensitive dye, Nile blue A. Chemical structures of NAS and basic and acidic forms of NBA is shown in Figure 1. NAS was synthesized in our laboratory according to the method in the literature [11]. Nile Blue chloride was supplied by Sigma Chemical $\mathrm{Co}$, and transformed to its basic form by extracting into benzene from its alkaline aqueous solution. Poly(methylmethacrylate) (PMMA) as a polymer matrix was used after reprecipitation. Other chemicals were commercially purchased and used without further purification. Sodium hydroxide $(\mathrm{NaOH})$ dissolved into methanol was added to the sample for keep the initial state of NBA basic. NBA base, $\mathrm{NAS}$, and $\mathrm{NaOH}$ was added in a chloroform solution of PMMA with a molar ratio of 1:2:1. and the obtained solution was cast onto a glass cover slip. The cast film was dried in air, followed by drying in vacuo.

The NSOM used in the present study was built in our laboratory, which is schematically illustrated in Figure 2. NSOM probes were fabricated by a chemical etching method followed by aluminum coating on the sidewalls [6]. The sample is mounted on the sample stage connected with a piezo scanner to scan the sample. The distance between the NSOM probe and the sample surface was controlled with a shear force technique $[12,13]$. An NSOM probe was attached to a small piezoelectric transducer which vibrates the probe. The vibration amplitude

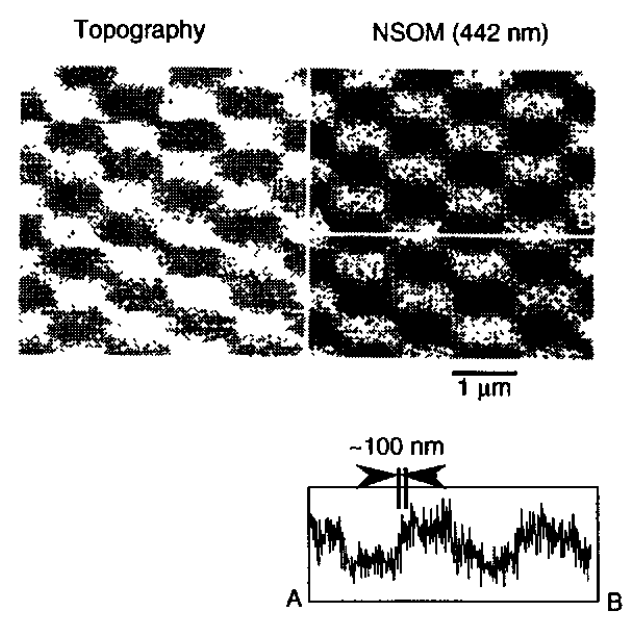

Figure 3 Simultaneously obtained topographic image (left-hand side) and corresponding NSOM image (right-hand side) of a chromium test pattern. Bright regions in the topographic image correspond to deposited chromium with an average thickness of $5 \mathrm{~nm}$. A cross-section along line AB in the NSOM image is also presented. 


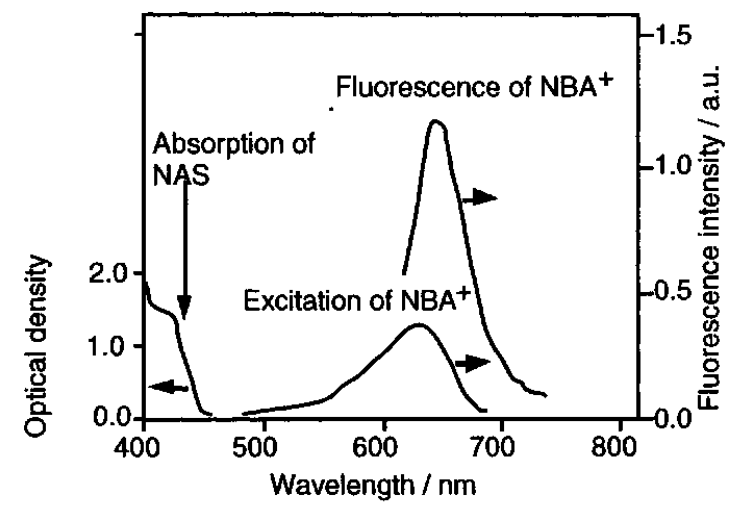

Figure 4 An absorption spectrum of NAS, and a fluorescence and its excitation spectra of NBA cation. The fluorescence was observed with an excitation wavelength of $600 \mathrm{~nm}$. The excitation spectrum was obtained by monitoring the fluorescence at $700 \mathrm{~nm}$.

of the probe is detected using an optical detection technique; a semiconductor laser (wavelength 670 $\mathrm{nm}$ ) was focused onto the side of the NSOM probe and scattered light is collected by a photodiode which generates a feedback signal. The light source for photoacid generation was a He-Cd laser with a wavelength of $442 \mathrm{~nm}$. The laser for detecting fluorescence from $\mathrm{NBA}^{+}$was a DCM dye laser at $620 \mathrm{~nm}$ which was pumped with an argon ion laser.

\section{Results and discussion}

In order to examine the spatial resolution of our NSOM, a chromium test pattern was measured, as shown in Figure 3 . This pattern has a period of 1 $\mu \mathrm{m}$, and the chromium thickness is approximately $5 \mathrm{~nm}$. The light source was a He-Cd laser with a wavelength of $442 \mathrm{~nm}$. The bright squares in the topographic image are areas coated with chromium at which light is blocked, and the dark squares are bare glass substrate through which visible light can pass. The contrast of the NSOM image is inverted compared to the topographic image. The crosssection of the NSOM image along the line AB is also presented in Figure 3. Stronger NSOM signal is observed at the area of the bare glass, and the spatial resolution of the NSOM is estimated from the sharpness of the start (or end) of the signal increase (or decrease) at the edges of the square. The sharpness was defined as the width corresponding to signal change from $10 \%$ to $90 \%$ of intensity maximum. Based on this definition, the spatial resolution of our NSOM system is evaluated to be about $100 \mathrm{~nm}$, twice as high as the diffraction limit.

An absorption spectrum of NAS, a fluorescence and its excitation spectra of NBA cation are displayed in Figure 4. From this figure it is observed that NAS has no absorption at $620 \mathrm{~nm}$ while NBA cation has a significant absorption there, indicating that the dye laser at $620 \mathrm{~nm}$ does not induce photoacid generation from NAS.

A film of PMMA doped with NAS and NBA was irradiated with a He-Cd laser at $442 \mathrm{~nm}$ for about 1 hours in order to induce photoacid generation from NAS. This was performed by positioning the NSOM probe at one point on the sample, at which the sample is illuminated by the light coming from the aperture of the NSOM probe. After the irradiation, the fluorescence from NBA cations was mapped using NSOM, as shown in Figure 5. The topographic image of the same area was obtained simultaneously. The circle shown in the upper right part in the topographic image indicates the area where the sample was irradiated with a $442 \mathrm{~nm}$ laser. In the corresponding area in the NSOM image, fluorescence signal originating from NBA cations is larger than in the other part, indicating that this area is acidified after the irradiation of $442 \mathrm{~nm}$ light. It can thus be concluded that a photoacid generation is induced in this area. The cross-section of the NSOM image along the line $A B$ is also displayed in Figure 5. In the cross-section there are two intensity peaks (left one and center one) in the fluorescence image, in addition to one corresponding to the irradiated area (right one). Such peaks are observed throughout this NSOM image, but their intensities are less than half of the intensity measured at the irradiated area, and there is no correlation between these peaks with the pits formed with NSOM probe. It can therefore be estimated
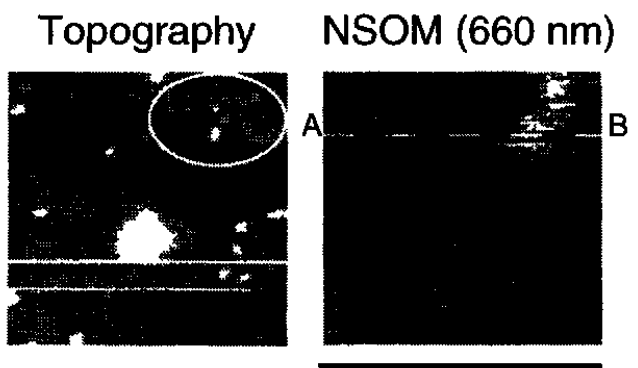

$15 \mu \mathrm{m}$

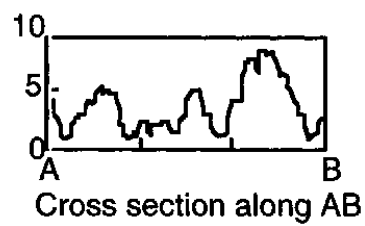

Figure 5 Simultaneously obtained topographic image (left-hand side) and corresponding fluorescent NSOM image (right-hand side) of a PMMA film doped with NAS and NBA. The film was exposed to $442 \mathrm{~nm}$ laser light from NSOM probe for 1 hour before obtaining image. The excitation source was a DCM dye laser at $620 \mathrm{~nm}$, and Fluorescence from NBA cations at 660 $\mathrm{nm}$ is mapped with NSOM. A cross-section along line $\mathrm{AB}$ in the NSOM image is also displayed. 

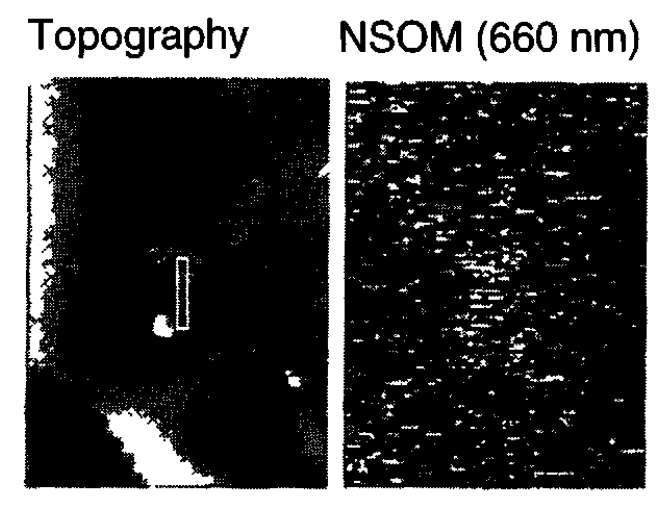

$12 \mu \mathrm{m}$

Figure $6 \mathrm{~A}$ rectangular pattern formed by the NSOM probe. The rectangle drawn in the topography indicates the irradiated area. The other conditions are same as those for Figure 5.

that such additional peaks originate from the noise of the system. There are some pits in the topographic image of Figure 5, indicating that the probe should have crashed against the sample surface. The sample is most severely damaged in the irradiated area at which the tip was placed for longer time than the other region of the sample. The pits are also observed in other area of the topography, but no significant fluorescent signal is observed there, indicating that the observed fluorescent signal is caused by photoacid generation during the irradiation, not by the mechanical contact by the tip. In the center of the topography a hill-like topographic feature with a diameter of about $3 \mu \mathrm{m}$ is observed. The reasons for the formation of such hill structures are not understood yet. A possible origin of this is a small sodium hydroxide crystal (for keeping the initial state of NBA basic) covered with polymer layers, or a bubble formed during the evaporation of the solvent from the film. At least, however, it can be concluded that this hill-like structure does not provide fluorescent signal, which is associated with the photoacid generation.

As shown in Figure 5, the tip position drifted during the photoirradiation. To estimate the broadening, a rectangular area with $0.3 \mu \mathrm{m}$ long in horizontal direction and $2 \mu \mathrm{m}$ in vertical direction was irradiated using NSOM probe, followed by mapping of the fluorescence from NBA cations. As shown in Figure 6, the drawn rectangle is smeared. In this case, the broadening of the edges of the pattern is approximately $3 \mu \mathrm{m}$. Considering the spatial resolution evaluated from Figure 3, this broadening value is not satisfactory. One of the most possible mechanisms for the broadening is the thermal drift of the microscope, The sample is scanned with the probe in two hours which was four times as long as that for obtaining Figure 3. Such mechanical drifts are often problematic in long time scanning in scanning probe microscopies, and they may be reduced by decreasing the size of the NSOM module.

To summarize, NSOM has been applied to the photoacid generation and to mapping the spatial distribution of photoacid in a polymeric solid. It is demonstrated that the photochemical products (in this study, photoacid) may be quantitatively estimated, and that simultaneously obtained topographic images will provide morphological information in addition to spectroscopic one. At present, the sample surface are damaged by the mechanical contact of the NSOM probe, and there is a considerable amount of thermal drift which makes the position control of the NSOM probe difficult. In order to estimate the diffusion coefficients of the photoacids in the polymer, the design of NSOM, including the size of NSOM unit, should be improved so that such damage and drifts should be avoided, and the results will be presented in the future study.

\section{Acknowledgments}

The authors acknowledge Ms. Mai Shigyo and Mr. Akira Yamakawa for their important contribution of synthesizing NAS.

\section{References}

[1] Ito H. IBM J. Res. Develop., 1997, 41, 69.

[2] MacDonald, A; Willson, C. G.; Frechet, M. J. Acc. Chem. Res., 1994, 27, 151.

[3] Yamakawa, A.; Horie, K. J. Photopolym. Sci \& Technol., 1999, 12, 297.

[4] Davis, M. M.; Hetzer, H. B.; Anal. Chem. 1966, 38,451 .

[5] Betzig, E.; Trautman, J. K. Science 1992, 257, 189-195.

[6] Ohtsu, M. ed."Near-Field Nano/Atom Optics and Technology", Springer-Verlag Tokyo, 1998.

[7] Bopp, M. A.; Meixner, A. J.; Tarrach, G.; Zschokke-Granacher, I.; Novotny, L. Chem. Phys. Lett. 1996, 263, 721.

[8] Ruiter, A. G. T.; Veerman, J. A.; Garcia-Parajo, M. F.; van Hulst, N. F. J. Phys. Chem. A 1997, 101,7318 .

[9] Jiang, Z. F. ; Ichihashi, J. ; Monobe, H. ; Fujihira, M. ; Ohtsu, M Opt. Commun, 1994, 106, 173.

[10] Machida, M.; Shigyo, M.; Yamakawa, A.; Osawa, H.; Horie, K. Tech. Dig. Sth International Conference on Near Field Optics and Related Techniquies, Shirahama, 1998, 297.

[11] Omote, T; Koseki, K.; Yamaoka, T. Macromolecules 1990, 23, 4788.103.

[12] Betzig, E.; Finn, P. L.; Weiner, J. S. Appl. Phys. Lett. 1992, 60, 2484.

[13] Toledo-Crow, R.; Yang, P. C.; Chen, Y.; VaezIravani, M. Appl. Phys. Lett. 1992, 60, 2957. 